[Vicino Oriente XXI (2017), pp. 273-277]

\title{
ROMA, IL CULTO DI ISIDE E SERAPIDE IN CAMPO MARZIO: ALCUNI AGGIORNAMENTI
}

\begin{abstract}
Alessandra Ten - Sapienza Università di Roma
This paper summarises some results arising from the research on the greatest Egyptian sanctuary in Rome, which was consecrated to Isis and Serapis and placed in the central Campo Marzio. The reading of Renaissance drawings and remains mentioned in that area made it possible to add a piece to the knowledge of that context which made a deep mark on the religious and urban history of the city.
\end{abstract}

Keywords: Rome; Egypt; Campo Marzio; temple of Isis and Serapis; sacred architecture

Com'è noto la localizzazione del più importante luogo sacro alle divinità egizie Iside e Serapide a Roma è un'acquisizione molto precoce nella storia degli studi archeologici. Essa fu dedotta essenzialmente sulla base dell'impressionante concentrazione di rinvenimenti architettonici e scultorei egizi ed egittizzanti compiuti nel tempo in tutta l'area attualmente racchiusa dall'asse Via del Seminario-Via S. Stefano del Cacco, rispettivamente a nord e a sud, e da quello costituito da via di S. Ignazio-Via del Gesù-Chiesa di S. Maria sopra Minerva a est e ad ovest ${ }^{1}$. Esiti più puntuali circa la configurazione e l'articolazione del complesso in questo settore furono acquisiti negli anni Quaranta del secolo scorso da Guglielmo Gatti che, grazie ad alcuni frammenti della pianta marmorea severiana e alle integrazioni ricavate dalla documentazione archivistica, attribuì in modo definitivo la pertinenza dei pochi resti ricadenti nell'area all'Iseo-Serapeo del Campo Marzio, noto dalle fonti letterarie ${ }^{2}$. Gli studi successivi hanno sempre accolto questa interpretazione; partendo dalle fonti iconografiche e dalle cronache dei rinvenimenti effettuati nel corso dei secoli, l'attenzione degli studiosi si è così spostata sulla definizione dei luoghi di culto veri e propri, sulla loro tipologia e dislocazione nell'ambito della vasta area attribuita al santuario, giungendo a conclusioni diverse anche in ordine ai suoi possibili modelli di origine ${ }^{3}$. Più recentemente lo studio diretto e la restituzione dei resti effettivamente conservati nel quadro topografico di riferimento hanno dimostrato la criticità di alcuni aspetti sostanziali nella ricostruzione proposta da G. Gatti: essi riguardano principalmente i due ingressi al grande organismo, l'arco di Camilliano e il cosiddetto arco di Giano alla Minerva, fondamentali per l'aggancio della ricostruzione alla topografia moderna. Gli approfondimenti dello studio su quest'ultima testimonianza, condotti dopo la scoperta e l'analisi dei suoi resti in uno scantinato del corrispondente palazzo moderno, restituiscono un quadro molto più articolato rispetto a quello prospettato nell'interpretazione tradizionale ${ }^{4}$. La collocazione, la denominazione e la ricostruzione dell'arco di Giano alla Minerva proposte da G. Gatti derivano, com'è noto, da uno schizzo realizzato da Antonio da Sangallo sicuramente dopo

1 Lanciani 1883. Per la rassegna complessiva dei rinvenimenti nell’area cfr. Malaise 1972, 112-245; Lembke 1994, 37-273. La sintesi dei rinvenimenti di importazione è in Lollio Barberi - Parola - Toti 1995.

2 Gatti 1942; 1943-1944; Carettoni et al. 1960, 97-102.

3 Su questi temi cfr. Malaise 1978, 683; Roullet 1972, 24, 30; Lembke 1994, 23, 25; Ensoli 1998, 413-417; Lollio Barberi - Parola - Toti 1995, 63-65.

4 Lanciani 1881, 279-280; Gatti 1942; De Maria 1988, 299-300; Lembke 1994, 183-184; Ten 2011, 735-736; 2015, 51-59; 2017; Attilia 2015.

ISSN 0393-0300

e-ISSN 2532-5159 
il 1515 (fig. 1) ${ }^{5}$. Non ci si è mai soffermati sul fatto che l'architetto fu attivo nei lavori di progettazione intrapresi per modificare la cappella absidale della chiesa di Santa Maria sopra Minerva, incarico al quale fu chiamato tra il 1530 e il $1540^{6}$. I resti della grande costruzione romana da lui riferita ad un arco quadrifronte erano e sono tuttora collocati in parte subito all'esterno dell'estremità meridionale del transetto della chiesa, una circostanza che spiega innanzitutto le ragioni che portarono l'architetto a rilevare queste strutture. Dello studio preliminare al progetto si conserva uno schizzo planimetrico misurato della cappella maggiore, realizzato dal fratello di Antonio, Giovanni Battista, per documentare lo stato di fatto $^{7}$. Osservando questo documento non si può fare a meno di notare come la distanza tra i pilastri di testata della cappella absidale sia molto vicina a quella annotata da Antonio da Sangallo per il grande spazio centrale del Giano (fig. 1): 48 palmi nell'abside, presi presumibilmente a partire dai rivestimenti, 49 nell'aula a crociera del Giano acanto ala Minerva, come riferiscono le glosse che accompagnano lo schizzo. Il rilievo del Peruzzi, realizzato poco tempo prima $^{8}$, si discosta leggermente da quello dei Sangallo, registrando una distanza di 47 palmi, ma anche in questo caso sussiste la possibilità che le misure tengano conto dei rivestimenti. La larghezza della navata centrale nella chiesa attuale è di $11,13 \mathrm{~m}$, molto vicina alla luce del fornice centrale $(11,06 \mathrm{~m})$ ricostruito da Gatti per l'ingresso occidentale all'Iseo-Serapeo. Se è difficile pensare ad un caso per la corrispondenza tra queste misure, è altrettanto difficile interpretare questa ricorrenza, tenendo conto soprattutto della prossimità fra le due strutture (distanti poco più di $18 \mathrm{~m}$ ) e della loro dislocazione sullo stesso asse. Partendo però dalla constatazione che il transetto insiste su murature antiche, isolate e descritte in occasione di uno studio capillare della chiesa $^{9}$, e che queste presentano caratteristiche molto simili per la tecnica edilizia a quelle rintracciate nello scantinato che ingloba i resti dell'arco di Giano, è forte la tentazione di immaginare una ripetizione dello stesso schema planimetrico, quindi una successione di grandi ambienti coperti a crociera, particolarmente sviluppati in elevato e disposti sullo stesso asse. L'eventualità di questa lettura configurerebbe un organismo estremamente imponente, sfuggito finora ai tentativi di ricostruire la fisionomia del grande santuario egizio a Roma; e acquisterebbero così un senso anche la testimonianza circa l'esistenza di un palatium Camilli, che ricorre già nella redazione più antica dei Mirabilia e che viene ribadita dall'Anonimo Magliabechiano, con l'indicazione del sito: Palatium Camilli fuit ubi nunc est arcus Camilliani retro Minervam ${ }^{10}$. Nei Mirabilia il termine Palatium distingue sempre organismi architettonici imponenti e nel caso in questione la natura e l'estensione dei resti sembra dare e, allo stesso tempo trovare, una spiegazione proprio in questa denominazione.

5 Antonio da Sangallo il Giovane, Uff. 1152; Bartoli 1917, vol. III, tav. CCLXVI, fig. 451, arch. 1152; Gatti 1943-1944, 137-150; De Maria 1988, 299-300; Lembke 1994, 183-184; Ten 2015, 51.

$6 \quad$ Palmerio - Villetti 1994, 34-38.

7 G.B. da Sangallo, Uff. 1661v A. Cfr. Palmerio - Villetti 1989, 122-132, fig. 23; Palmerio - Villetti 1994, 3435, fig. 25.

$8 \quad$ Palmerio - Villetti 1989, 125-126, fig. 20.

$9 \quad$ Palmerio - Villetti 1989, 74-76.

10 Su questo aspetto cfr. Roncaioli 1979, 86. 


\section{BIBLIOGRAFIA}

ATTILIA, L.

2015 1871-1875: Scoperta e distruzione del cd. Giano alla Minerva in Campo Marzio - La storia e le carte: F. FILIPPI (ed.), Campo Marzio. Nuove ricerche, Roma 2015, pp. 31-40.

BARTOLI, A.

1917 I monumenti antichi di Roma nei disegni degli Uffizi di Firenze, Roma 1917.

CARetToni, G. - Colini, A.M. - CozzA, L. - GatTi, G.

1960 La pianta marmorea di Roma antica. Forma urbis Romae, 2 voll., Roma 1960.

DE MARIA, S.

1988 Gli archi onorari di Roma e dell'Italia Romana, Roma 1988.

ENSOLI, S.

1998 L'Iseo e Serapeo del Campo Marzio con Domiziano, Adriano e i Severi: l'assetto monumentale e il culto legato con l'ideologia e la politica imperiali: N. BonACASA - M.C. NARO - E.C. Portale - A. Tullio (a cura di), L'Egitto in Italia dall'antichità al Medioevo. Atti del III Congresso Internazionale Italo-Egiziano. Roma, CNR - Pompei, LANCIANI, R. 13-19 Novembre 1995, Roma 1998, pp. 407-438.

1881 Relazione sull'isolamento del Pantheon: Notizia degli Scavi di Antichità (1881), pp. 255294.

1883 L'Iseum et Serapeum della Regione IX: Bullettino della Commissione Archeologica LEMBKE, $\mathrm{K}$. Comunale di Roma XI (1883), pp. 33-60.

1994 Das Iseum Campense in Rom. Studie über den Isiskult unter Domitian, Heidelberg 1994.

Lollio BARBeri, G. - PAROla, G. - Toti, M.P.

$1994 \quad$ Le Antichità Egiziane di Roma Imperiale, Roma 1994.

MALAISE, $M$.

1972 Inventaire préliminaire des documents égyptiens découverts en Italie (Études GATTI, G. préliminaires aux religions orientales dans l’Empire romain 21), Leiden 1972.

1942 Un ignoto monumento adrianeo nel Campo Marzio: L’Urbe VII.1 (1942), pp. 2-14.

1943-1944 Topografia dell'Iseo Campense: Rendiconti della Pontificia Accademia Romana di Archeologia XX (1943-1944), pp. 117-163.

PALmerio, G. - Villetti, G.

1989 Storia edilizia di S. Maria sopra Minerva, 1275-1870, Roma 1989.

1994 Santa Maria sopra Minerva in Roma. Notizie dal cantiere, Roma 1994.

RONCAIOLI, C.

1979 L'arco di «Camilliano» e il «Cacco» di S. Stefano nell’ Iseo e Serapeo del Campo Marzio: ROULLET, A Giornale Italiano Filologia n.s. X (1979), pp. 81-96.

1972 The Egyptian and Egyptianizing Monuments of Imperial Rome (Études préliminaires aux religions orientales dans l'Empire romain 20), Leiden 1972.

TEN, A.

2011 Considerazioni preliminari su un settore del Campo Marzio centrale: Scienze dell'Antichità 17 (2011), pp. 733-737.

2015 Sulla ricostruzione del contesto topografico antico nel Campo Marzio centrale: F. FILIPPI (ed.), Campo Marzio. Nuove ricerche, Roma 2015, pp. 41-75. 
2017 Roma 1870, divergenze tra la nuova pianificazione urbana e la tutela del patrimonio archeologico: cronaca di un caso controverso in Campo Marzio: M. AYARZAGÜEnA SANZ - G. Mora - J. SAlas Álvarez (eds.), 150 Años de Historia de la Arqueologìa: Teorìa y método de una disciplina (Memorias de la Sociedad Española de Historia de la Arqueología III), Arganda del Ray 2017, pp. 883-892. 


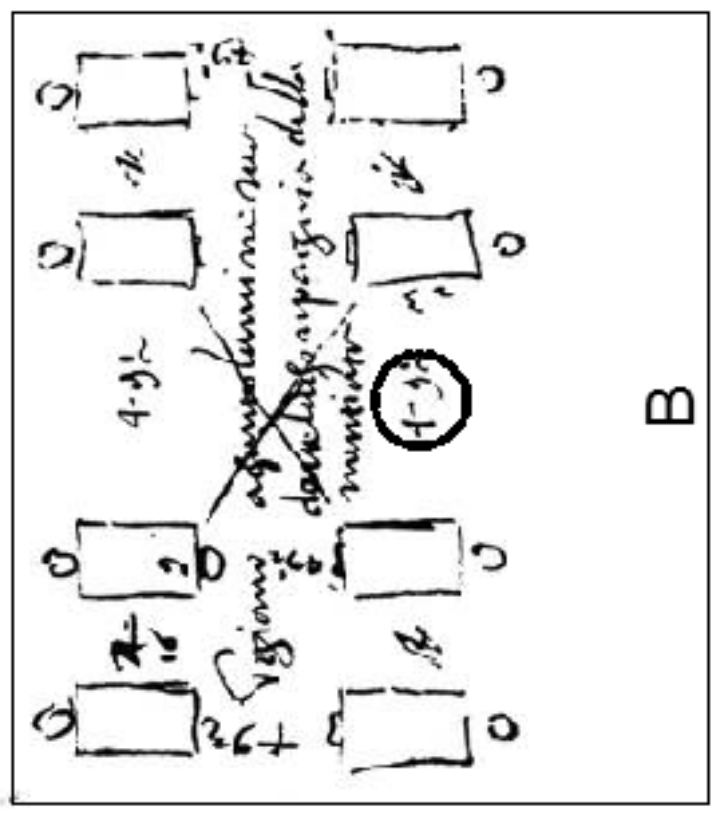

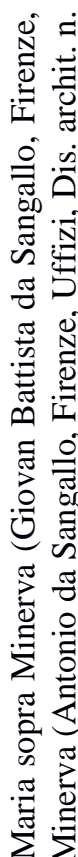

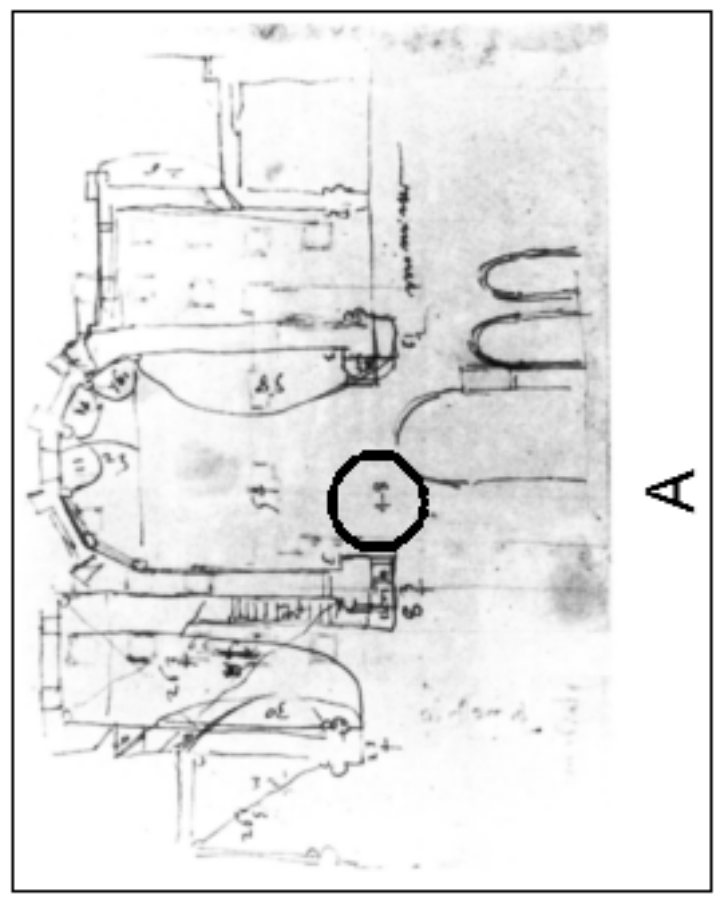

䒕 $\frac{\pi}{\pi}$

๘

.

은

त्र

तี

훙

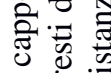

$\pi .7$

चे $\ddot{\theta}$

\& $\ddot{\tau} \bar{q}$

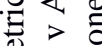

킁

즌

व

운

武氖

安究宁

- $\hat{\text { กิ }}$

汇苛先 